\title{
Circ_0000260 Regulates the Development and Deterioration of Gastric Adenocarcinoma with Cisplatin Resistance by Upregulating MMPII via Targeting MiR-I29-5p
}

This article was published in the following Dove Press journal: Cancer Management and Research

\section{Shicheng Liu \\ Miao Wu \\ Mengyin Peng}

Department of Gastrointestinal Surgery, The Second People's Hospital of Yibin, Yibin, Sichuan, 644000, People's Republic of China
Correspondence: Miao Wu Department of Gastrointestinal Surgery, The Second People's Hospital of Yibin, No. 96 North Street, Yibin, Sichuan, People's Republic of China Tel +8683I-82550II

Email feohdoctor@I26.com
Background: Cisplatin (CDDP) plays a vital role in the treatment of advanced gastric adenocarcinoma (GAC); however, the development of chemoresistance depletes the overall benefit of CDDP. This study harbored the aim to investigate the role of a novel circular RNA (circRNA), circ 0000260, in DDP-resistant GAC and provide a potential mechanism to explain its function. Methods: The morphology of tumor tissues and normal tissues was observed by hematoxylin-eosin (HE) staining. The isolated exosomes were observed and examined using transmission electron microscopy (TEM) and nanoparticle tracking analysis (NTA). The expression of circ_0000260, miR-129-5p and matrix metalloproteinase 11 (MMP11) mRNA was measured by quantitative real-time polymerase chain reaction (qRT-PCR). The protein levels of CD63, CD81, fibronectin, vitronectin and MMP11 were detected by Western blot. Cell viability, colony formation, cell apoptosis, migration, invasion and cell adhesion were monitored by cell counting kit-8 (CCK-8) assay, colony formation assay, flow cytometry assay, scratch assay, transwell assay and cell adhesion assay, respectively. The interaction between miR-129-5p and circ_0000260 or MMP11 predicted by bioinformatics analysis was verified by dual-luciferase reporter assay. Animal experiments were performed in nude mice to explore the role of circ 0000260 in vivo.

Results: The expression of circ_0000260 was promoted in tumor tissues and serum-derived exosomes of GAC patients, and circ_0000260 expression in CDDP-resistant tumor tissues was higher than that in CDDP-sensitive tumor tissues. Circ_0000260 knockdown lessened CDDP chemoresistance, suppressed cell proliferation, migration, invasion and adhesion, and induced apoptosis. In mechanism, circ_0000260 regulated the expression of MMP11 by targeting miR-129-5p. MiR-129-5p inhibition could reverse the functions of circ_0000260 knockdown, and MMP11 knockdown could also reverse the effects of miR-129-5p inhibition. Besides, circ_0000260 knockdown attenuated CDDP resistance during tumor growth in vivo by regulating the expression of miR-129-5p and MMP11.

Conclusion: Circ_0000260 regulated CDDP chemoresistance of GAC by promoting MMP11 expression via targeting miR-129-5p.

Keywords: circ_0000260, gastric adenocarcinoma, CDDP, miR-129-5p, MMP11

\section{Introduction}

Gastric cancer is a common malignant tumor in all digestive tract cancers, and is divided into gastric adenocarcinoma (GAC) and gastric squamous cell carcinoma (GSCC) according to the histopathological features. ${ }^{1,2}$ GAC, also termed as 
stomach adenocarcinoma (STAD), is the main subtype of gastric cancer, ${ }^{2}$ accounting for $90-95 \%$ of all gastric cancer cases. Most GAC cases were diagnosed as advanced, leading to an unsatisfied prognosis, with an average 5-year survival rate of $29 \% .{ }^{3}$ Chemotherapy is a common way to prevent the recurrence of local disease and transfer risk after surgery. ${ }^{4}$ However, the overall benefit of chemotherapy is largely limited because of the development of drug resistance during treatment, especially multi-drug resistance, which seriously interferes with the effect of chemotherapy, leading to metastasis and recurrence of GAC. ${ }^{4,5}$ Identification of the molecular factors associated with chemoresistance in GAC will help to broaden the eyes to understand the development and treatment of this cancer.

With the advance of sequencing technology, multiple types of non-coding RNAs, including circular RNAs (circRNAs) and microRNAs (miRNAs), were demonstrated to be aberrantly expressed in gastric cancer tissues or cells. ${ }^{6,7}$ Based on this, numerous circRNAs and miRNAs were screened and functionally identified to be involved in diagnostic, prognosis value, and progression of gastric cancer. ${ }^{89}$ However, there is still a lack of research on the chemoresistance of circRNAs. Previous studies reported that circRNA-AKT3 expression was increased in cisplatin (CDDP)-resistant tissues and cells of gastric cancer, and circRNA-AKT3 contributed to DNA damage repair and inhibited gastric cancer cell apoptosis by regulating CDDP resistance. ${ }^{10}$ Similarly, circRNA-PVT1 was abundantly existed in paclitaxel-resistant gastric cancer cells and conferred paclitaxel resistance. ${ }^{11}$ Here, circ_0000260, deriving from structural maintenance of chromosomes 3 (SMC3) mRNA, is largely functionally unknown. Whether circ_0000260 plays a role in the progression of cancer resistance is worth investigating.

Accumulating evidence uncovered that circRNAs served as "molecular sponges" of targeted miRNAs to suppress miRNA expression and function in cancers. ${ }^{10,11}$ Following this mechanism, we found that miR-129-5p was predicted as a target of circ_0000260 by bioinformatics. Although the role of miR-129-5p in gastric cancer has been widely exhibited, ${ }^{12,13}$ its mechanism of action remains to be further elucidated, such as the relationship between miR-129-5p and circ_0000260.

It is well known that miRNAs generally play their functions by directly binding to the $3^{\prime}$ untranslated region (3'UTR) of certain mRNAs. ${ }^{14}$ Similarly in miR-129-5p, for example, miR-129-5p restoration repressed migration, invasion and other malignant behaviors of gastric cancer cells by binding to interleukin-8 or COL1A1. ${ }^{12,13}$ Here, matrix metalloproteinase 11 (MMP11) is interestingly predicted to be targeted by miR-129-5p, and its expression is elevated in GAC tissues by Gene Expression Profiling Interactive Analysis (GEPIA), suggesting that MMP11 is likely involved in miR-129-5p regulatory pathway. However, this hypothesis has not been verified in the current research.

Briefly, in the present study, we investigated the expression of circ_0000260 in CDDP-resistant and CDDPsensitive GAC tissues and cells. Besides, functional analyses were performed to explore the role of circ_0000260 on CDDP chemoresistance in cellular activities in vitro and tumor growth in vivo. Moreover, the circ_0000260/ miR-129-5P/MMP11 pathway involved in CDDPresistance development was identified to provide a potential mechanism of circ_0000260, aiming to provide a scheme for chemoresistant therapy of GAC.

\section{Materials and Methods}

\section{Clinical Tissues and Serum Samples}

A total of 27 GAC patients and 27 healthy volunteers were recruited from The Second People's Hospital of Yibin. GAC tumor tissues $(n=27)$ and adjacent normal tissues $(\mathrm{n}=27)$ were removed from GAC patients, and blood samples were also collected from GAC patients and healthy volunteers. Serum samples were obtained from blood samples by centrifugation. GAC patients received treatment with standard CDDP-based therapeutic regimens after surgery and subsequently were classified into two groups, including CDDP-sensitive and CDDP-resistant groups, according to the relapse or progression within 6 months after the last cisplatin-based chemotherapy. CDDPresistance was defined as tumor recurrence or relapse, and CDDP-sensitivity was defined as no tumor recurrence or relapse. Importantly, informed consent was signed by all participants prior to sample collection. All tissues and blood samples were stored at $-80^{\circ} \mathrm{C}$ conditions. This study was implemented with the approval of the Ethics Committee of The Second People's Hospital of Yibin. The research has been carried out in accordance with the World Medical Association Declaration of Helsinki.

\section{Hematoxylin-Eosin (HE) Staining}

Tumor tissues and normal tissues were fixed with formaldehyde and dehydrated with a concentration gradient of 
alcohol $(70 \%, 80 \%, 90 \%, 95 \%$, and $100 \%)$ and then probed with xylene. Subsequently, tissues were subjected to wax immersion and paraffin embedding, and tissues were sliced into $4-\mu \mathrm{m}$-thick sections. The sections were baked at $60^{\circ} \mathrm{C}$ and then stained with HE solution (Sigma, St. Louis, MO, USA). Next, the sections were dehydrated with alcohol and sealed with neutral gum. At last, the sections were observed by an optical microscope $(\times 100$; Olympus, Tokyo, Japan).

\section{Exosome Isolation}

Exosomes were isolated from serum samples using the exoEasy Maxi kit (Qiagen, Duesseldorf, Germany) according to the instruction.

\section{Transmission Electron Microscopy (TEM)}

For morphology investigation of exosomes, exosome pellet was placed on formvar carbon-coated 200-mesh copper electron microscopy grids and incubated for 5 mins at room temperature, followed by $1 \%$ uranyl acetate staining for $1 \mathrm{~min}$ at room temperature. The grid was washed with PBS, dried at room temperature and next observed under a TEM H7500 (Hitachi, Tokyo, Japan).

\section{Nanoparticle Tracking Analysis (NTA)}

The size distribution and concentration of exosomes were determined using NTA. In brief, exosomes were exposed to $1 \mathrm{~mL}$ PBS and mixed evenly, then the diluted exosomes were injected into the NanoSight NS300 instrument (Malvern, UK), and the particle size was automatically tracked according to Brownian motion and the diffusion coefficient. The NTA measurement conditions were set as 25 frames per second, with measurement time of $60 \mathrm{~s}$.

\section{Quantitative Real-Time Polymerase Chain Reaction (qRT-PCR)}

Total RNA was isolated and purified using the RNeasy Mini Kit (Qiagen). Then, RNA was reversely transcribed into cDNA using the miScript reverse transcription kit (Qiagen), and next cDNA was used for qRT-PCR amplification using the miScript SYBR-Green PCR kit (Qiagen) according to the protocols. The relative fold change was assessed using the $2^{-\Delta \Delta \mathrm{CT}}$ method, with $\beta$-actin or U6 as an internal reference. The primer sequences were exhibited as below:

circ_0000260: F, 5'-AGATCGAGACCCAGCAAAGG-3' and R, 5'-TGACTTGGTCACCTTAGGCAT-3'; $\beta$-actin: F, $5^{\prime}$ -
CCAAGGCCAACCGCGAGAAGATGAC-3' and R, 5'AGGGTACATGGTGGTGCCGCCAGAC-3'; miR-129-5p: F, 5'-GCGGCTTTTTGCGGTCTGG-3' and R, 5'-GTGCA GGGTCCGAGGT-3'; U6: F, 5'-CTCGCTTCGGCAGCAC A-3' and R, 5'-AACGCTTCACGAATTTGCGT-3'; MMP11: F, 5'-AAGAGGTTCGTGCTTTCTGG-3' and R, 5'CCATGGGAACCGAAGGAT-3';

\section{Western Blot}

The procedures of Western blot analysis were implemented following the description previously described. ${ }^{15}$ The proteins stained on the PVDF membranes were blocked in skim milk and incubated with the primary antibodies, including anti-CD63 (ab134045; Abcam, Cambridge, MA. USA), anti-CD81 (ab109201; Abcam), antifibronectin (ab45688; Abcam), anti-vitronectin (ab113700; Abcam), anti-MMP11 (ab53143; Abcam) and anti- $\beta$-actin (ab8227; Abcam) at $4{ }^{\circ} \mathrm{C}$ for $12 \mathrm{hrs}$, followed by the incubation with the secondary antibody (ab205718; Abcam) at room temperature for $2 \mathrm{hrs}$.

\section{Cell Lines and Cell Culture}

GAC cell lines (HGC27 and AGS) were purchased from EK-Bioscience (Shanghai, China) and cultured in matched complete medium (Item Number: CC-Y1228M and CCY1045M; EK-Bioscience). HGC27 and AGS cells were exposed to continuously increasing concentrations of CDDP $(0.5-20 \mu \mathrm{M})$ for over 6 months to establish CDDPresistant cell lines (HGC27/CDDP and AGS/CDDP). ${ }^{16}$

\section{Cell Counting Kit-8 (CCK-8) Assay}

CCK-8 assay was implemented to determine the viability of HGC27/CDDP and AGS/CDDP cells after CDDP treatment, or CDDP resistance detection, HGC27, AGS, HGC27/CDDP and AGS/CDDP cells treated with different concentrations of $\operatorname{CDDP}(0,1,2,4,8,12,16,20$ and $24 \mu \mathrm{M})$ were planted into 96 -well plates $\left(3 \times 10^{3}\right.$ cells/ well), followed by the addition of $10 \mu \mathrm{L}$ CCK- 8 reagent (Beyotime, Shanghai, China) after culturing for 48 h. Then, the absorbance value at $450 \mathrm{~nm}$ was detected under a microplate reader (Bio-Rad, Hercules, CA, USA). The IC50 of CDDP was the point of CDDP concentration when cell survival rate dropped to $50 \%$. For cell proliferation, cells seeded in 96-well plates were treated with $12 \mu \mathrm{M}$ CDDP and cultured for $12,24,48$ or $72 \mathrm{~h}$, and then cell viability was examined by CCK-8 assay as mentioned above. Each group contained five replicate wells. 


\section{Cell Transfection}

We used small interference RNA (siRNA) to mediate circ_0000260 and MMP11 knockdown. The oligonucleotides, including siRNA targeting circ_0000260 (si-circ\#1, \#2 and \#3), siRNA targeting MMP11 (si-MMP\#1, siMMP\#1 and si-MMP\#1) and siRNA negative control (siNC), were all synthesized by Ribobio (Guangzhou, China). MiR-129-5p mimics (miR-129-5p), miR-129-5p inhibitors (anti-miR-129-5p) and corresponding control (miR-NC and anti-NC) were also purchased from Ribobio. For circ_0000260 overexpression, overexpression plasmid pCD5-ciR-circ_0000260 (circ_0000260) and matched control (Vector) were constructed by Geneseed (Guangzhou, China). HGC27/CDDP and AGS/CDDP cells were transfected with these oligonucleotides or plasmids using Lipofectamine 3000 (Invitrogen, Carlsbad, CA, USA).

\section{Colony Formation Assay}

Cells were trypsinized and seeded onto 6-well plates (300 cells per well) in triplicate. Cells in plates were placed at $37^{\circ}$ $\mathrm{C}$ conditions containing $5 \% \mathrm{CO}_{2}$ to allow colony growth. After maintaining for 15 days, cells were fixed and stained with crystal violet (Sigma) for $5 \mathrm{~min}$. The number of colonies was counted under a microscope (Olympus).

\section{Flow Cytometry Assay}

Flow cytometry was carried out to analyze cell apoptosis using the Annexin V-FITC Apoptosis Detection Kit (Invitrogen). HGC27/CDDP and AGS/CDDP cells were collected at $48 \mathrm{~h}$ post-transfection and trypsinized. Cells were then washed with PBS, and resuspended in $200 \mu \mathrm{L}$ binding buffer (density: $3 \times 10^{5}$ cells). Subsequently, $5 \mu \mathrm{L}$ Annexin V-FITC was added to incubate cells for $10 \mathrm{~min}$. Then, cells were washed and resuspended in $200 \mu \mathrm{L}$ binding buffer, followed by the treatment of $10 \mu \mathrm{L}$ Propidium Iodide (20 $\mu \mathrm{g} / \mathrm{mL}$ ) for $5 \mathrm{~min}$. The apoptotic cells were detected using a flow cytometer (BD Biosciences, San Jose, CA, USA).

\section{Scratch Assay}

Scratch assay was performed to assess cell migration. After $24 \mathrm{~h}$ transfection, HGC27/CDDP and AGS/CDDP cells planted into 6-well plates were grown to confluence and scratched using a sterile $200 \mu \mathrm{L}$ pipette tip to create a wound. Cells were washed with PBS to remove detached cells and incubated in the serum-free complete growth medium. Photos $(100 \times)$ were taken after incubation for $24 \mathrm{~h}$ to monitor wound healing. The rate of wound closure was assessed using the Image $\mathrm{J}$ software (National Institutes of Health, Bethesda, MA, USA).

\section{Transwell Assay}

To investigate cell invasion, transfected HGC27/CDDP and AGS/CDDP cells in $500 \mu \mathrm{L}$ serum-free culture medium were transferred into the top of Matrigel-coated transwell chambers (BD Biosciences). The lower chambers were filled with a $500-\mu \mathrm{L}$ culture medium containing $10 \%$ FBS. After $24 \mathrm{~h}$, cells remaining on the upper surface were removed with cotton wool, and cells in the lower surface were fixed and stained with crystal violet (Sigma) for $5 \mathrm{mins}$, followed by the observation under a microscope (100×; Olympus).

\section{Cell Adhesion Assay}

Transfected HGC27/CDDP and AGS/CDDP cells $\left(4 \times 10^{4}\right.$ cells) were seeded into 24-well plates, which were precoated with Matrigel (BD Biosciences). The plates were placed at $37^{\circ} \mathrm{C}$ conditions containing $5 \% \mathrm{CO}_{2}$, and corresponding medium containing non-adhered cells was discarded at 30-, 60-, and 90-min intervals. Besides, cells were washed twice with PBS to remove potential loosely attached cells. Then, the adherent cells were analyzed using CCK-8 assay as mentioned above.

\section{Bioinformatics Analysis}

Bioinformatics tools, including circBank (http://www.cir cbank.cn/) and starBase (http://starbase.sysu.edu.cn/), were utilized to analyze the potential target miRNAs of circ_0000260. The potential target mRNAs of miR-129-5p were analyzed by starBase, miRWalk (http://mirwalk. umm.uni-heidelberg.de/). Besides, the top 100 of mRNAs which were highly expressed in GAC tissues were obtained from Gene Expression Profiling Interactive Analysis (GEPIA: http://gepia.cancer-pku.cn/).

\section{Dual-Luciferase Reporter Assay}

The wild-type sequence fragment of circ 0000260 or MMP11 3'UTR (containing original miR-129-5p binding site) and the mutant sequence fragment of circ_0000260 or MMP11 3'UTR (containing mutated miR-129-5p binding site) were amplified and cloned into pmirGLO reporter plasmid (Promega, Madison, WI, USA), and the fusion plasmids were named as WT-circ_0000260, MUT-circ _0000260, WT-3'UTR MMP11 and MUT-3'UTR MMP11. HGC27/CDDP and AGS/CDDP cells were cotransfected with WT-circ_0000260, MUT-circ 
0000260, WT-3'UTR MMP11 or MUT-3'UTR MMP11 and miR-129-5p or miR-NC using Lipofectamine 3000. The luciferase activities were measured at $48 \mathrm{~h}$ posttransfection using Dual-Luciferase Reporter Assay System (Promega) in line with the protocols.

\section{Animal Experiments}

BALB/c mice (6-8 weeks old, female) were purchased from JunKe Biology (Nanjing, China) and housed in pathogen-free conditions. For the subcutaneously injected tumor model, $2 \times$ $10^{6}$ AGS cells were transfected with short hairpin RNA targeting circ_0000260 (sh-circ) or control (sh-NC), which was synthesized by BrainVTA Co., Ltd (Wuhan, China) and packaged into lentiviral vector (LV). All mice were randomly divided into 4 groups ( $\mathrm{n}=5$ per group). AGS cells with shcirc transfection were implanted into nude mice from any 2 groups, and AGS cells with sh-NC transfection were implanted into nude mice from another 2 groups. After inoculation, one group of mice injected with sh-circ- or sh-NC-transfected AGS cells were intraperitoneally administered with CDDP at a dose of $5 \mathrm{mg} / \mathrm{kg}$ according to body weight once a week for 4 weeks. During this period, tumor volume (length $\times$ width $^{2} / 2$ ) was assessed every 4 days. After 28 days, all mice were killed, and tumor tissues were harvested for weight measurement and other analyses. All experimental procedures were done with the approval of the Animal Care and Use of Committee of The Second People's Hospital of Yibin. Animal studies were performed in compliance with the ARRIVE guidelines and the Basel Declaration. All animals received humane care according to the National Institutes of Health (USA) guidelines.

\section{Statistical Analysis}

All data from at least three independent experiments were processed using SPSS 21.0 software (IBM Corp., Armonk, NY, USA) and displayed as mean \pm standard deviation. Analysis of variance (ANOVA) was used for multiple comparisons, and Student's $t$-test was adopted for comparisons in two groups. Correlation analysis was performed using the Spearman correlation test. A $p$-value of $<0.05$ was considered statistically significant.

\section{Results}

Circ_0000260 Was Upregulated in GAC Tissues and Serum-Derived Exosomes of GAC Patients

HE staining of sections prepared from samples of GAC tissues and adjacent normal tissues is shown in Figure 1A.
In these clinical specimens, the expression of circ 0000260 was significantly increased in GAC tumor tissues $(n=27)$ compared with that in normal tissues $(\mathrm{n}=27)$ (Figure 1A). Meanwhile, exosomes were isolated from serum samples from GAC patients and healthy volunteers. The representative morphology of exosomes was observed by TEM and characterized by typical lipid bilayer membraneencapsulated nanoparticles, and the data from NTA showed that the isolated exosomes had an approximate size of 130 $\mathrm{nm}$ or $110 \mathrm{~nm}$ from GAC patients and healthy volunteers, respectively (Figure 1B). The data from Western blot presented that the expression of exosome-related proteins, including CD63 and CD81, was reinforced in the isolated exosomes (Figure 1C). Besides, the expression of circ 0000260 in serum-derived exosomes from GAC patients was higher than that in serum-derived exosomes from healthy volunteers (Figure 1D). These GAC patients were classified into two groups, including CDDP-sensitive group and CDDP-resistant group. We discovered that the expression of circ_0000260 was notably elevated in serumderived exosomes from the CDDP-resistant group compared with that from CDDP-sensitive group (Figure 1E). These data suggested that circ_0000260 was highly expressed in GAC tumor tissues and serum-derived exosomes, and high expression of circ_0000260 might be associated with CDDP resistance.

\section{Circ_0000260 Was Highly Expressed in CDDP-Resistant GAC Cells, and Circ_0000260 Knockdown Inhibited Proliferation, Migration, Invasion and Adhesion of CDDP-Resistant GAC Cells} The value of IC50 of these induced CDDP-resistant HGC27 $(15.76 \mu \mathrm{M})$ and AGS $(18.13 \mu \mathrm{M})$ cells was significantly higher than their parental HGC27 $(7.98 \mu \mathrm{M})$ and AGS $(7.06 \mu \mathrm{M})$ cells (Figure 2A). Besides, cell viability analysis showed that HGC27/CDDP and AGS/CDDP cells were less responsive to CDDP treatment compared to their parental cells (Figure 2B). These data suggested that CDDP-resistant GAC cells were successfully generated. The data from qRTPCR presented that the expression of circ_0000260 was enhanced in HGC27 and AGS cells compared with that in GES-1 cells, and the expression of circ_0000260 in HGC27/ CDDP and AGS/CDDP cells was also notably higher than that in HGC27 and AGS cells, respectively (Figure 2C). Here, siRNA was used to mediate circ_0000260 knockdown. The data showed that the expression of circ_0000260 was 
A

B

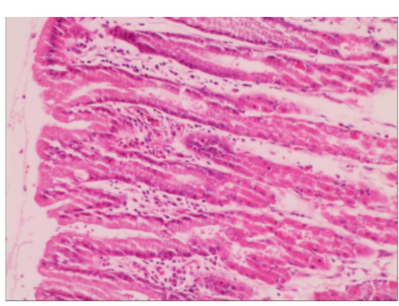

Normal

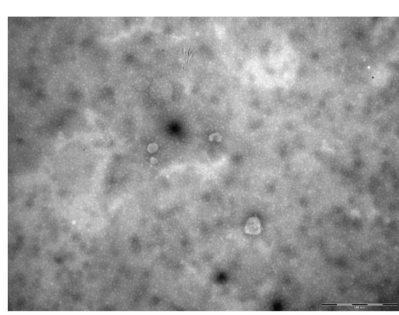

Healthy

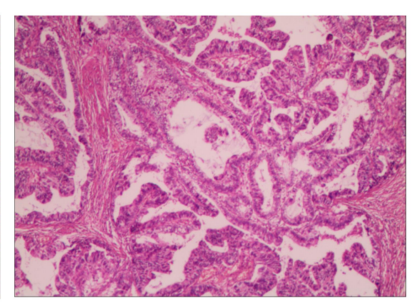

Tumor

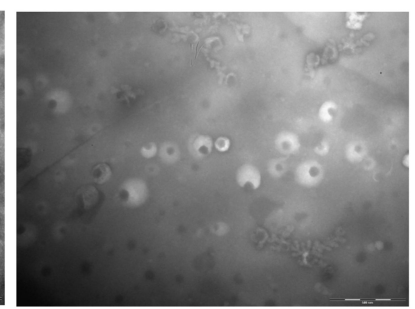

GAC
C

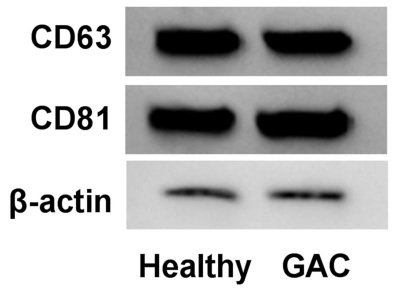

D

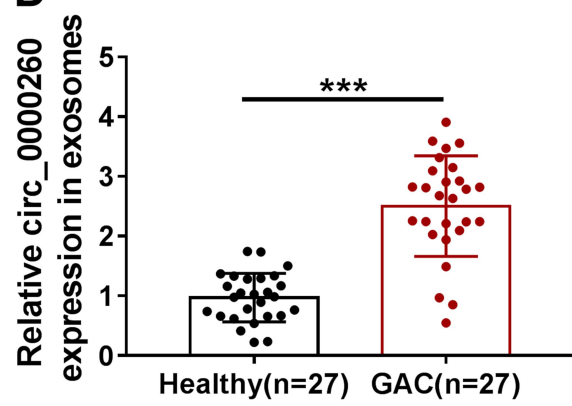

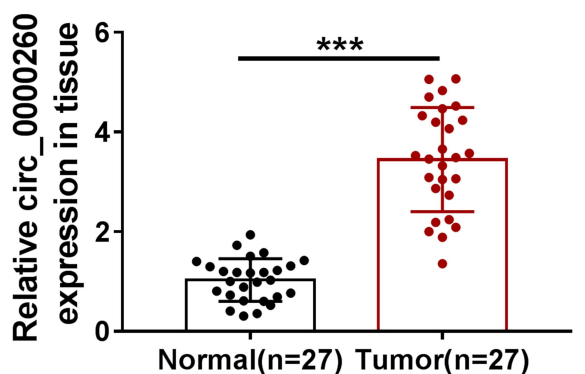

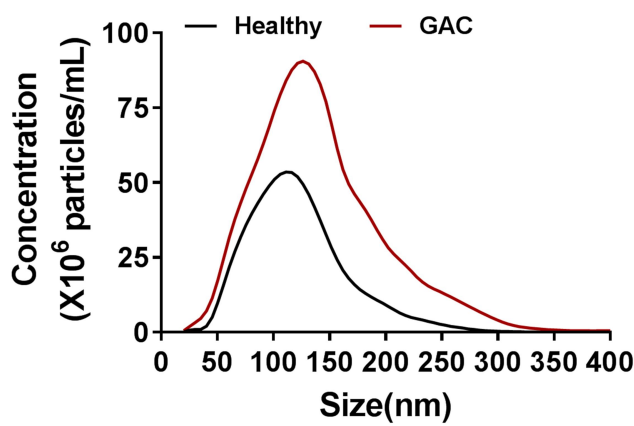

E

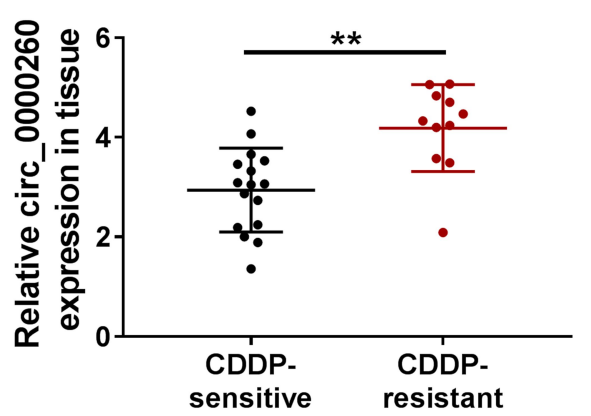

Figure I Circ_0000260 was highly regulated in GAC tissues and serum-derived exosomes. (A) The tissue sections of GAC tissues and adjacent normal tissues were observed by $\mathrm{HE}$ staining, and the expression of circ_0000260 in clinical tissues was detected by qRT-PCR. (B) The phenotype and size distribution of exosomes were identified by TEM and NTA. (C) The expression of exosomes-related markers was measured by Western blot. (D) The expression of circ_0000260 in serum-derived exosomes from GAC patients and healthy volunteers was detected by qRT-PCR. (E) The expression of circ_0000260 in CDDP-sensitive and CDDP-resistant GAC tissues was detected by qRT-PCR. $* * P<0.01$, $* * * P<0.001$.

strikingly decreased in HGC27/CDDP and AGS/CDDP cells transfected with si-circ\#1, si-circ\#2 and si-circ\#3, and sicirc\#2 induced the lowest expression of circ_0000260 (Figure 2D). Thus, si-circ\#2 was selected for subsequent analysis to minimize the off-target effects of siRNA. In function, CDDP treatment suppressed cell proliferation, and si-circ\#2 transfection significantly further reduced the proliferation of HGC27/CDDP and AGS/CDDP cells relative to si-NC transfection (Figure 2E). Meanwhile, circ_0000260 knockdown notably impaired the ability of colony formation (Figure 2F). As expected, circ_0000260 knockdown notably promoted the apoptotic rate of HGC27/ CDDP and AGS/CDDP cells (Figure 2G). Scratch assay showed that circ_0000260 knockdown led to a lower rate of wound closure, indicating that circ_0000260 knockdown blocked the ability of migration in HGC27/CDDP and AGS/ CDDP cells (Figure 2H). Transwell assay presented that circ_0000260 knockdown lessened the number of invaded cells, meaning that circ_0000260 knockdown blocked cell invasion (Figure 2I). Moreover, cell adhesion assay displayed that circ_0000260 knockdown suppressed the capacity of cell adhesion in HGC27/CDDP and AGS/CDDP cells (Figure 2J), and circ_0000260 knockdown resulted in a decrease of the expression of adhesion-related markers, including fibronectin and vitronectin, which also proved that circ_0000260 knockdown blocked cell adhesion (Figure $2 \mathrm{~K})$. These findings indicated that circ_0000260 knockdown enhanced CDDP chemosensitivity and thus suppressed a series of malignant behaviors of CDDP-resistant HGC27 and AGS cells. 

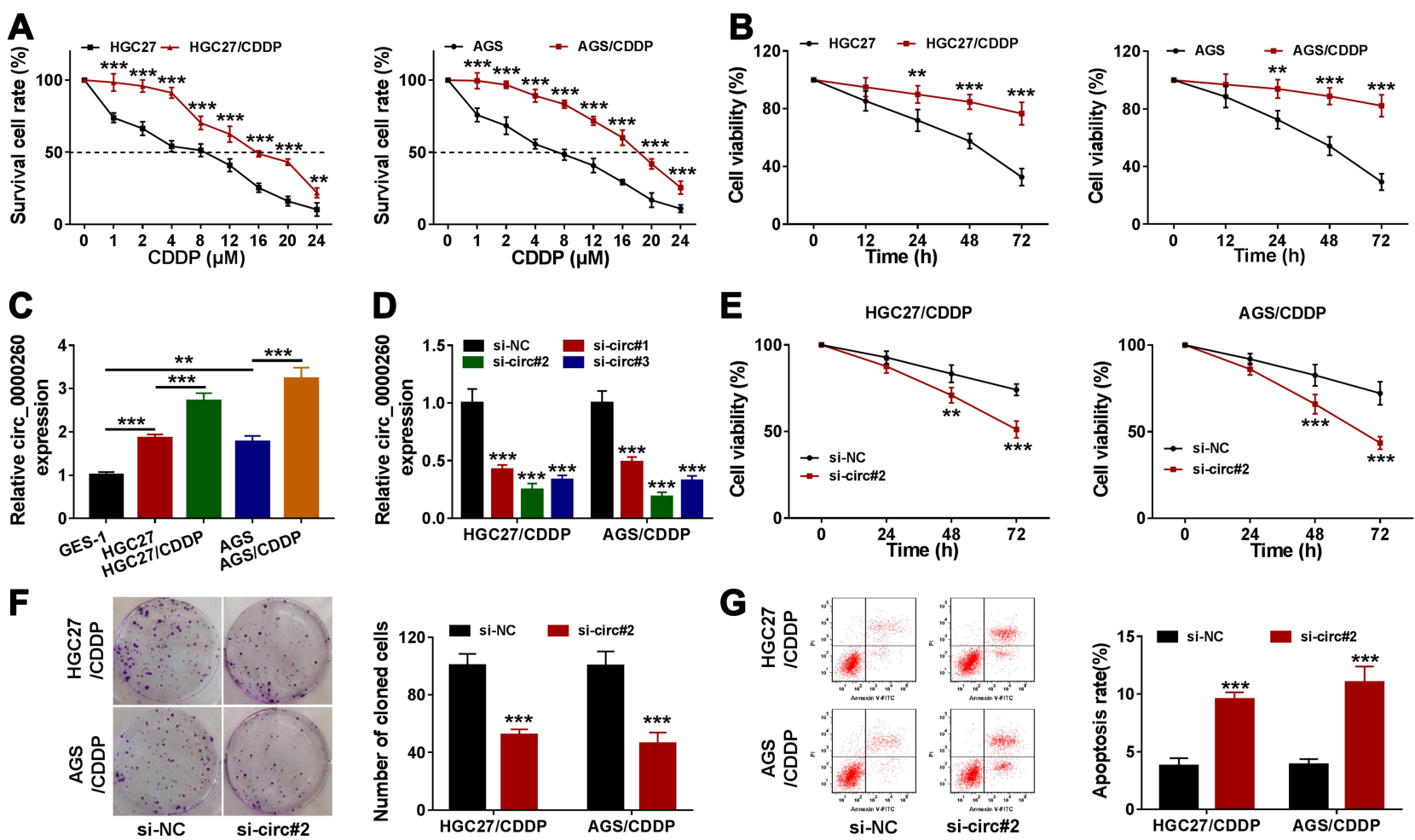

H
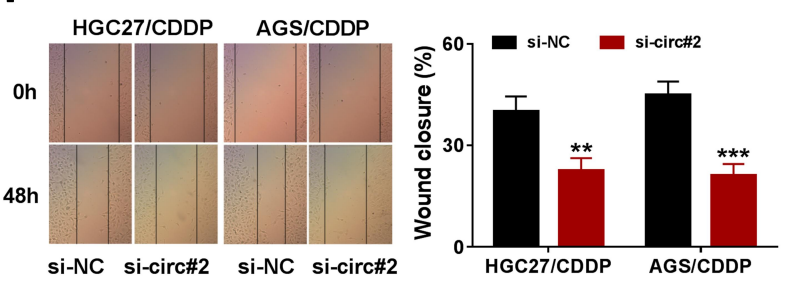

I
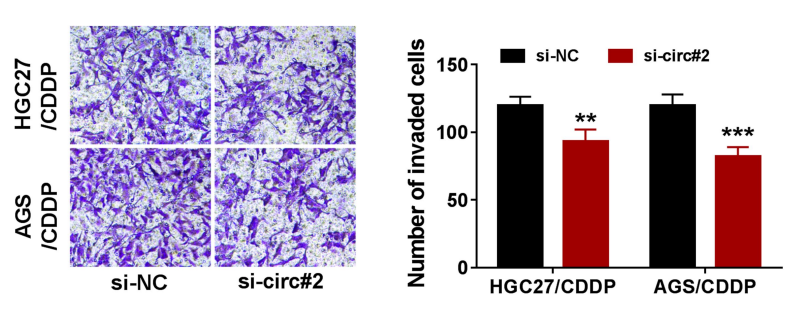

$\mathbf{J}$

$\mathbf{K}$
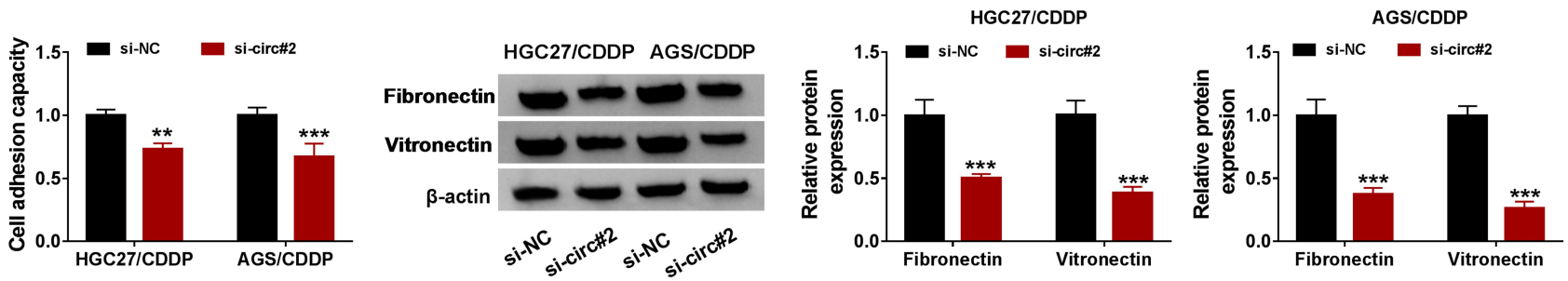

Figure 2 Circ_0000260 knockdown alleviated CDDP chemoresistance, thus blocking the malignant behaviors of HGC27/CDDP and AGS/CDDP cells. (A) The difference of cell survival in HGC27/CDDP, AGS/CDDP and their parental cells was monitored using CCK-8 assay with the treatment of different concentrations of CDDP. (B) Cell proliferation of HGC27/CDDP and HGC27 cells after the treatment of CDDP was assessed by CCK-8 assay. (C) The expression of circ_0000260 in HGC27/CDDP, AGS/ CDDP and their parental cells was detected by qRT-PCR. (D) The efficiency of siRNA was examined by qRT-PCR. In HGC27/CDDP and AGS/CDDP cells transfected with si-circ\#2 or si-NC, (E) cell proliferation was assessed by CCK-8 assay. (F) The ability of colony formation was investigated by colony formation assay. (G) Cell apoptosis was monitored by flow cytometry assay. (H and $\mathbf{I})$ Cell migration and cell invasion were determined by scratch assay and transwell assay, respectively. (J) Cell adhesion capacity was monitored using cell adhesion assay. (K) Cell adhesion-related proteins were quantified by Western blot. $* * P<0.0$ I, $* * * P<0.00 \mathrm{I}$.

\section{Circ_0000260 Targeted MiR-I29-5p to Suppress the Expression of MiR-129-5p}

To explore a potential mechanism of circ_0000260 action, we screened and identified several target miRNAs of circ_0000260. Bioinformatics analysis showed that three miRNAs, including miR-982-5p, miR-361-3p and miR$129-5 p$, were simultaneously identified by circBank and
starBase, and further analysis presented that the expression of miR-129-5p was highly increased in circ_0000260downregulated cells (Figure 3A). Hence, miR-129-5p of interest was used for the following analyses. The expression of miR-129-5p was remarkably declined in GAC tumor tissues $(n=27)$ compared with that in normal tissues $(n=27)$ (Figure 3B). The expression of miR-129-5p in GAC tissues 
A

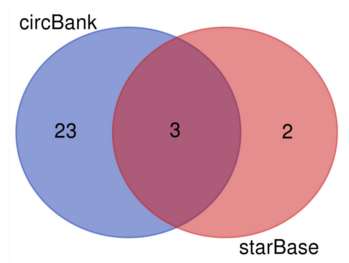

D
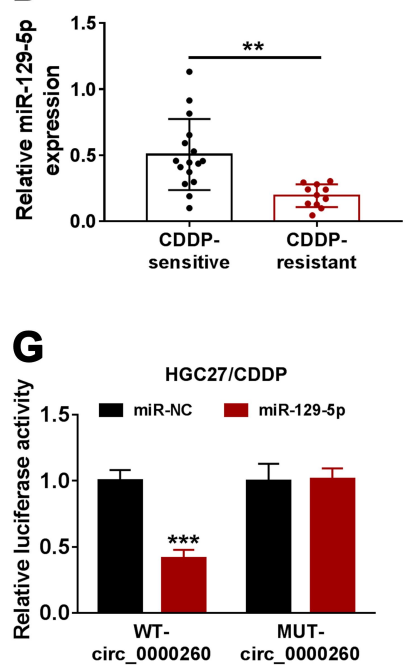

B

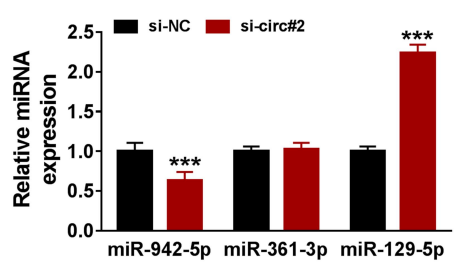

E
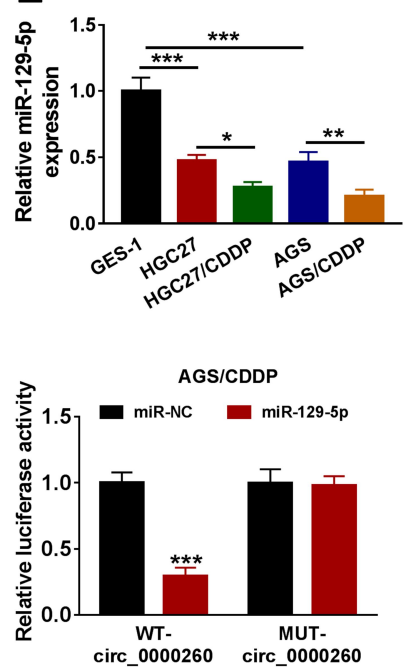

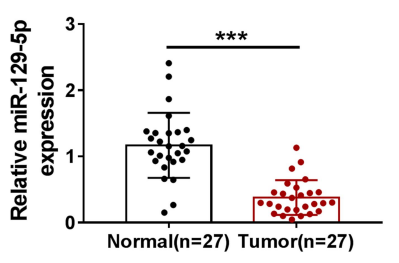

F
C

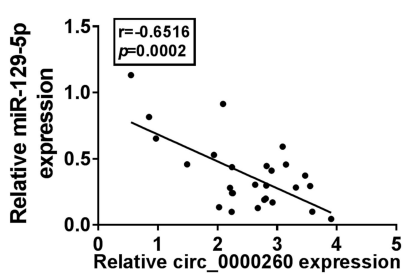

WT-circ_0000260 5' cucgacuugaauuGCAAAAAG 3' | | | | | | | |

hsa-miR-129-5p 3' cguucgggucuggCGUUUUUC 5'

MUT-circ_0000260 5' cucgacuugaauuAUCCCCCA 3'

Figure 3 MiR-129-5p was a target of circ_0000260. (A) The potential miRNAs targeted by circ_0000260 were predicted by circBank and starBase, and then the expression of miR-942-5p, miR-36I-3p and miR-129-5p in si-circ\#2-transfected cells was detected by qRT-PCR. (B) The expression of miR-I29-5p in GAC tumor tissues and adjacent normal tissues was measured by qRT-PCR. (C) The expression correlation between miR-129-5p and circ_0000260 in GAC tissues was analyzed by Spearman correlation analysis. (D) The expression of miR-129-5p in CDDP-resistant or CDDP-sensitive GAC tissues was detected by qRT-PCR. (E) The expression of miR-I29-5P in HGC27/ CDDP, AGS/CDDP, HGC27 and AGS cells was detected by qRT-PCR. (F) MUT-circ_0000260 sequence was designed according to WT-circ_0000260 sequence to conduct dual-luciferase reporter assay. (G) The interaction between circ_0000260 and miR-129-5p was verified by dual-luciferase reporter assay. (H) The efficiency of circ_0000260 overexpression was examined using qRT-PCR. (I) The expression of miR-129-5p in HGC27/CDDP and AGS/CDDP cells with circ_0000260 overexpression or knockdown was detected by $\mathrm{qRT}-\mathrm{PCR}$. $* P<0.05, * * P<0.01$, $* * * P<0.001$.

was negatively correlated with circ_0000260 expression (Figure 3C). In addition, we noticed that the expression of miR-129-5p in CDDP-resistant GAC tissues was lower than that in CDDP-sensitive GAC tissues (Figure 3D). The expression of miR-129-5p was also notably decreased in HGC27 and AGS cells compared to GES-1 cells, and miR129-5p expression was lower in HGC27/CDDP and AGS/ CDDP cells compared with that in HGC27 and AGS cells, respectively (Figure 3E). Next, we designed the mutant sequence fragment of circ_0000260 according to its wildtype sequence to perform dual-luciferase reporter assay (Figure 3F). The results showed that miR-129-5p and WTcirc_0000260 cotransfection markedly diminished luciferase activity in HGC27/CDDP and AGS/CDDP cells compared to miR-NC (Figure 3G), suggesting that miR-129-5p was a target of circ_0000260. The efficiency of circ_0000260 overexpression was available because the expression of circ_0000260 was strikingly enhanced in HGC27/CDDP and AGS/CDDP cells transfected with circ_0000260
(Figure 3H). The expression of miR-129-5p was notably impaired in HGC27/CDDP and AGS/CDDP cells with circ_0000260 overexpression but notably reinforced in cells with circ_0000260 knockdown (Figure 3I), suggesting that circ_0000260 negatively regulated miR-129-5p expression.

\section{MiR-I29-5p Inhibition Reversed the Effects of Circ_0000260 Knockdown to}

\section{Recover the Malignant Effects of HGC27/ CDDP and AGS/CDDP Cells}

MiR-129-5p was a target of circ_0000260, and we deduced that circ_0000260 regulated CDDP chemoresistance by modulating miR-129-5p. The expression of miR-129-5p was substantially decreased in HGC27/CDDP and AGS/CDDP cells transfected with anti-miR-129-5p compared to anti-NC (Figure 4A). Subsequently, HGC27/CDDP and AGS/CDDP cells were transfected with si-circ\#2, si-NC, si-circ\#2+antimiR-129-5p or si-circ\#2+anti-NC to monitor whether miR- 

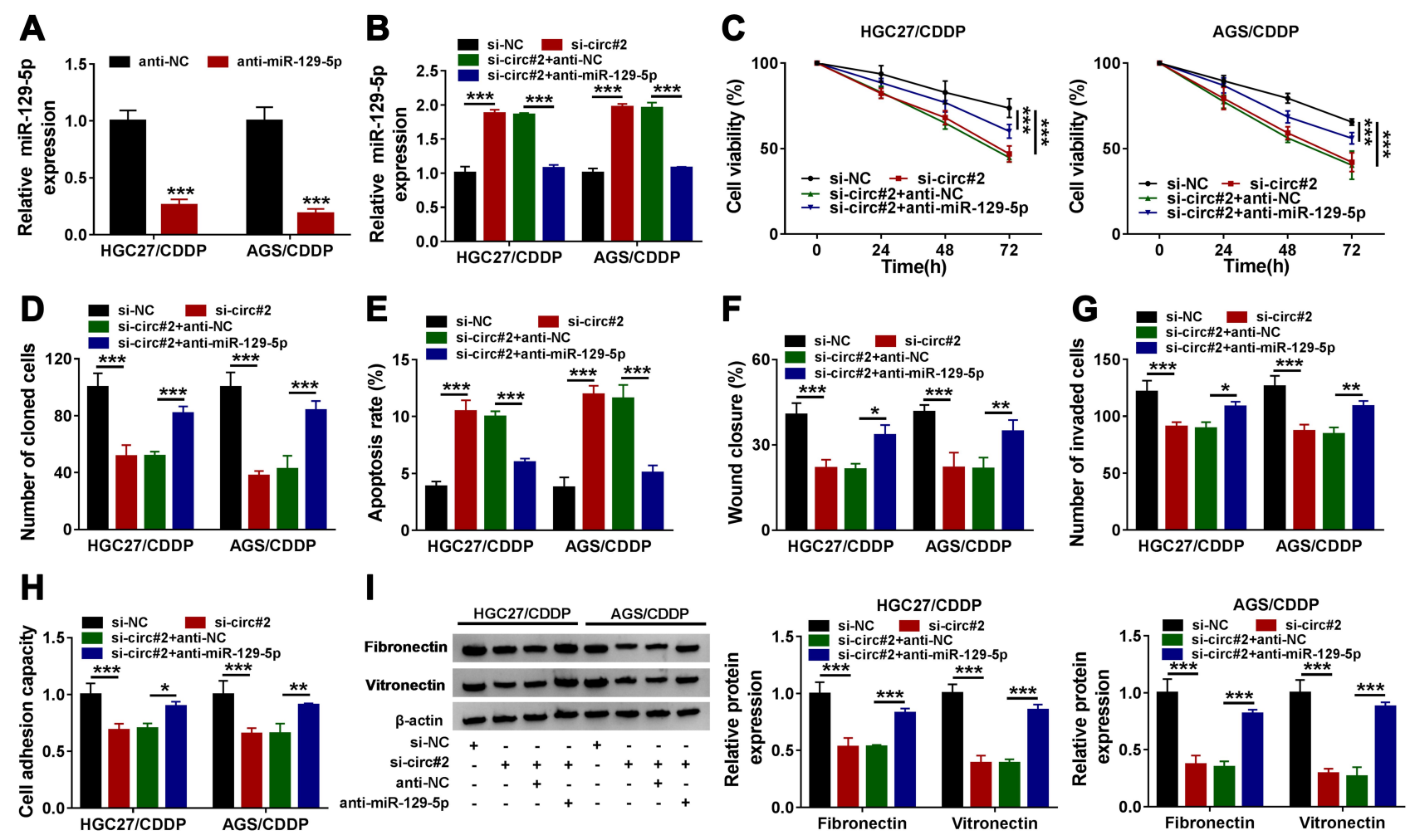

Figure 4 Circ_0000260 regulated CDDP chemoresistance of HGC27/CDDP and AGS/CDDP cells by targeting miR-I29-5p. (A) The efficiency of miR-I29-5p inhibitor was examined by $\mathrm{qRT}$-PCR. HGC27/CDDP and AGS/CDDP cells were next transfected with si-circ\#2, si-NC, si-circ\#2+anti-miR-I29-5P or si-circ\#2+anti-NC. In these transfected cells, (B) Cell proliferation of HGC27/CDDP and HGC27 cells after the treatment of CDDP was assessed by CCK-8 assay. (C) cell proliferation was monitored using CCK-8 assay. (D) Colony formation ability was assessed by colony formation assay. (E) Cell apoptosis was monitored by flow cytometry assay. (F and $\mathbf{G})$ Cell migration and cell invasion were assessed by scratch assay and transwell assay, respectively. (H) Cell adhesion capacity was detected by cell adhesion assay. (I) The expression of fibronectin and vitronectin was quantified by Western blot. $* P<0.05, * * p<0.01$, $* * * P<0.001$.

129-5p inhibition could rescue the role of circ_0000260 knockdown. The expression of miR-129-5p promoted in sicirc\#2-transfected cells was notably declined in si-circ\#2+antimiR-129-5p-transfected cells (Figure 4B). Transfected HGC27/CDDP and AGS/CDDP cells were treated with CDDP. The results showed that miR-129-5p inhibition recovered cell proliferation and colony formation ability which were blocked by circ_0000260 knockdown (Figure 4C and D). While, circ_0000260 knockdown-induced cell apoptosis was suppressed by miR-129-5p inhibition (Figure 4E). Scratch assay and transwell assay indicated that cell migration and invasion blocked by circ_0000260 knockdown were largely promoted by miR-129-5p knockdown (Figure $4 \mathrm{~F}$ and $\mathrm{G}$ ). Moreover, cell adhesion capacity weakened in si-circ\#2-transfected HGC27/CDDP and AGS/CDDP cells was recovered in si-circ\#2+anti-miR-129-5p-transfected cells (Figure $4 \mathrm{H}$ ), and the expression of fibronectin and vitronectin was also lessened in si-circ\#2-transfected HGC27/CDDP and AGS/CDDP cells but restored in si-circ\#2+anti-miR-129-5ptransfected cells (Figure 4I). The above evidence supported that miR-129-5p inhibition weakened CDDP chemosensitivity and recovered CDDP-resistant cell malignant behaviors which were blocked by circ_0000260 knockdown.

\section{MiR-I29-5p Bound to MMPII 3'UTR and Degraded MMPII Expression}

To further monitor whether circ_0000260 functioned through a "ceRNA" mechanism, the potential miRNAs targeted by miR-129-5p were identified. As shown in Figure 5A, a total of seven miRNAs, as miR-129-5p targets, were not only predicted by starBase and miRWalk but also detected in GEPIA, and the expression of MMP11 was significantly impaired in cells with miR-129-5p enrichment. Besides, GEPIA database introduced that the expression of MMP11 was aberrantly elevated in stomach adenocarcinoma (STAD) tissues $(n=408)$ compared to normal tissues $(n=211)$, which was verified in our clinical GAC tissues (Figure $5 \mathrm{~B}$ ). The expression of MMP11 was negatively correlated with miR129-5p expression in GAC tissues (Figure 5C). The data from the protein level of MMP11 also showed that MMP11 was upregulated in GAC tumor tissues (Figure 5D). Moreover, the expression of MMP11 at both mRNA and 
A

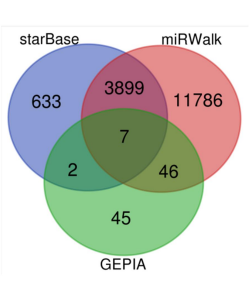

C

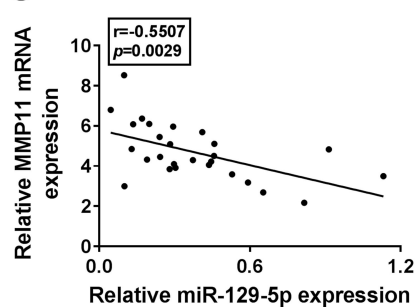

G

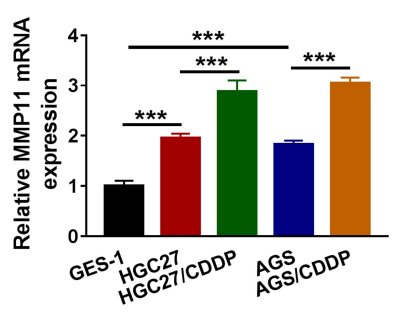

J

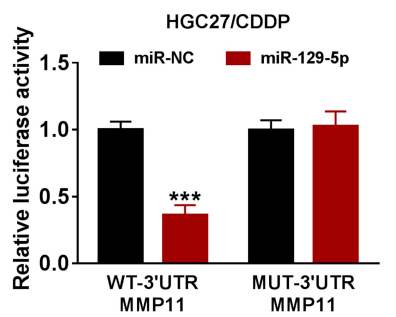

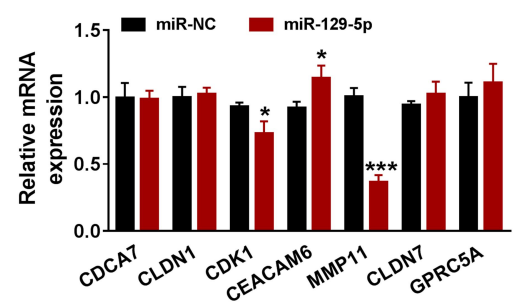

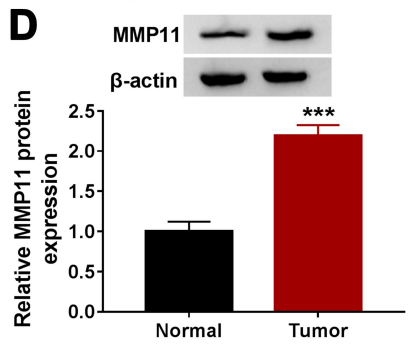

H
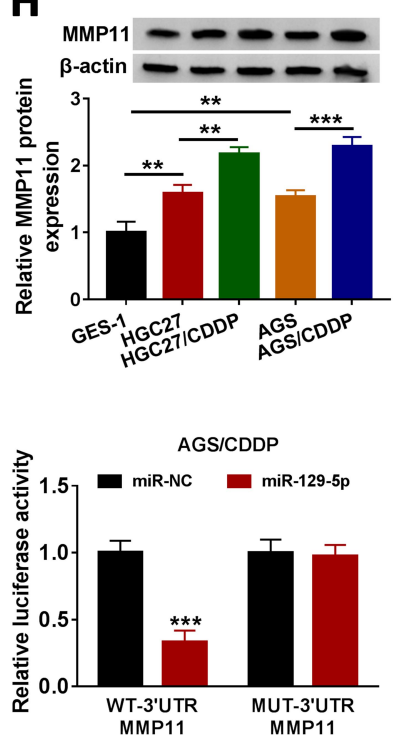

B

MMP11 expression of GEPIA
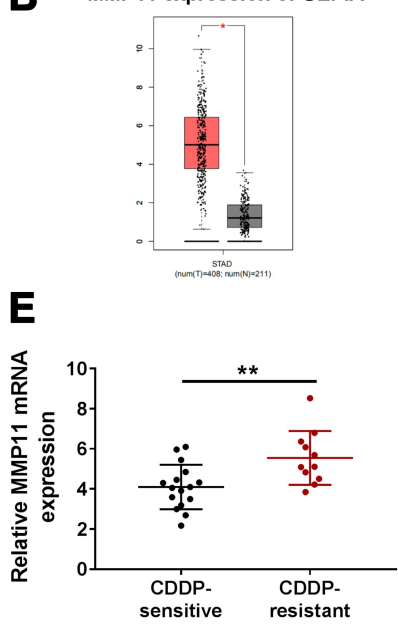

I
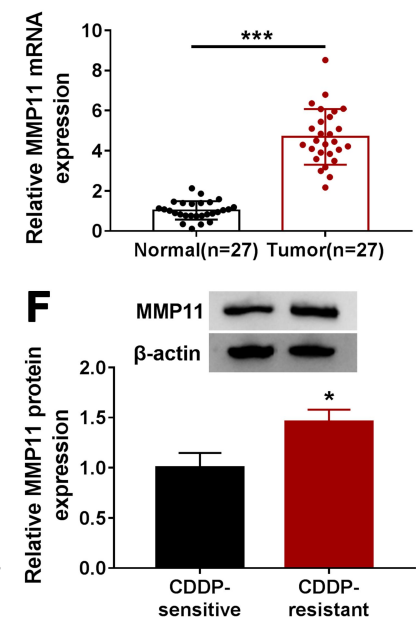

K

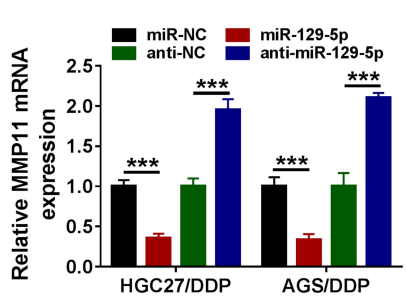

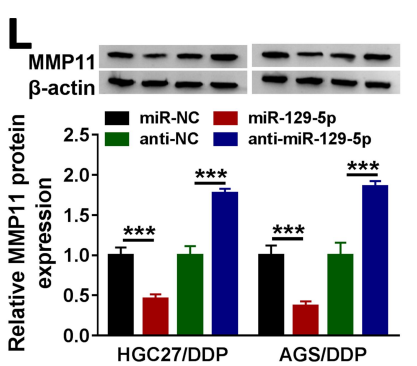

Figure 5 MMPII was a target of miR-129-5p and was upregulated in GAC tissues. (A) Venn diagram was depicted to screen mRNAs detected among starBase, miRWalk and GEPIA database, and their expression in HGC27/CDDP and AGS/CDDP cells transfected with miR-129-5P or miR-NC was detected by qRT-PCR. (B) The expression of MMPII in GAC and normal tissues was obtained from GEPIA database, and MMPII expression was also measured in clinical tissues by qRT-PCR. (C) The correlation between MMPII expression and miR-129-5p expression in GAC tissues was analyzed by Spearman's test. (D) The expression of MMPII expression in tumor tissues and normal tissues was detected by Western blot. (E and $\mathbf{F}$ ) The expression of MMPII in CDDP-sensitive and CDDP-resistant GAC tissues was detected by qRT-PCR and Western blot. (G and $\mathbf{H}$ ) The expression of MMPII in CDDP-sensitive and CDDP-resistant GAC cells was detected by qRT-PCR and Western blot. (I) MUT-MMPI I 3'UTR sequence was designed according to WT-MMPII 3'UTR sequence. (J) The relationship between miR-I29-5p and MMPII was verified by dual-luciferase reporter assay. ( $\mathbf{K}$ and $\mathbf{L}$ ) The expression of MMPII in HGC27/CDDP and AGS/CDDP cells with miR-129-5p overexpression or knockdown was detected by qRT-PCR and Western blot. $* P<0.05, * * P<0.01, * * * P<0.001$.

protein levels was relatively higher in CDDP-resistant tumor tissues than that in CDDP-sensitive tumor tissues (Figure 5E and F). Similarly, the expression of MMP11 at both mRNA and protein levels was elevated in HGC27 and AGS cells compared with that in GES-1 cells, and the expression of MMP11 was relatively higher in HGC27/CDDP and AGS/ CDDP cells compared with that in HGC27 and AGS cells, respectively (Figure 5G and $\mathrm{H}$ ). Next, according to the original sequence of MMP11 3'UTR containing miR-129-5p binding site, the mutant sequence of MMP11 3'UTR was designed (Figure 5I). Dual-luciferase reporter assay presented that the luciferase activity was strikingly declined in HGC27/CDDP and AGS/CDDP cells transfected with miR129-5p and WT-3'UTR MMP11 (Figure 5J). The expression of MMP1 at both mRNA and protein levels was remarkably impaired in HGC27/CDDP and AGS/CDDP cells with miR$129-5 p$ restoration but strengthened in cells with miR-129-5p inhibition (Figure $5 \mathrm{~K}$ and $\mathrm{L}$ ). These data verified that miR129-5p directly bound to MMP11 and suppressed MMP11 expression. 


\section{MiR-I29-5p Inhibition Promoted CDDP Chemoresistance and Thus Induced HGC27/CDDP and AGS/CDDP Cell Malignant Development, While MMPI I Knockdown Reversed These Effects}

We next explored whether miR-129-5p played functions by suppressing MMP11. Likewise, siRNA was used to mediate MMP11 knockdown, and the expression of MMP11 was strikingly decreased in HGC27/CDDP and AGS/CDDP cells transfected with si-MMP11\#1, siMMP11\#2 and si-MMP11\#3, and si-MMP11\#3 induced the lowest expression of MMP11 (Figure 6A and B). Thus, si-MMP11\#3 was selected for subsequent analysis to minimize the off-target effects of siRNA. The expression of MMP1 was enhanced in anti-miR-129-5ptransfected $\mathrm{HGC} 27 / \mathrm{CDDP}$ and AGS/CDDP cells but repressed in anti-miR-129-5p+si-MMP11\#3-transfected cells (Figure 6C and D). In function, miR-129-5p inhibition weakened the response of HGC27/CDDP and AGS/ CDDP cells to CDDP, which MMP11 knockdown partly enhanced the response of HGC27/CDDP and AGS/CDDP cells to CDDP (Figure 6E). The ability of colony formation in HGC27/CDDP and AGS/CDDP cells was strengthened by anti-miR-129-5p transfection but inhibited by anti-miR-129-5p+si-MMP11\#3 transfection (Figure 6F). Cell apoptosis was suppressed by miR-129-5p inhibition but recovered by MMP11 knockdown (Figure 6G). Cell migration and cell invasion were promoted in HGC27/ CDDP and AGS/CDDP cells transfected with anti-miR -129-5p but repressed in cells transfected with anti-miR $-129-5 p+s i-M M P 11 \# 3$ (Figure 6H and I). Moreover, the capacity of cell adhesion was heightened in HGC27/ CDDP and AGS/CDDP cells transfected with anti-miR -129-5p but repressed in cells transfected with anti-miR $-129-5 p+$ si-MMP11 $\# 3$, and this result was further verified by the expression of fibronectin and vitronectin (Figure $6 \mathrm{~J}$ and $\mathrm{K}$ ). These data suggested that miR-129-5p inhibition reduced CDDP chemosensitivity and aggravated malignant behaviors of HGC27/CDDP and AGS/CDDP cells by regulating MMP11.

\section{Circ_0000260 Functioned as a ceRNA to Regulate MMPI I Expression by Targeting MiR-I29-5P}

We detected that the expression of MMP11 was significantly declined in HGC27/CDDP and AGS/CDDP cells transfected with si-circ\#2 compared to si-NC, while the expression of MMP11 was largely recovered in HGC27/ CDDP and AGS/CDDP cells transfected with si-circ\#2 +anti-miR-129-5p compared to si-circ\#2+anti-NC at both mRNA and protein levels (Figure 7A and B), suggesting that circ_0000260 sponged miR-129-5p to regulate the expression of MMP11.

\section{Circ_0000260 Knockdown Enhanced CDDP Chemosensitivity to Further Block}

\section{Tumor Growth in vivo}

Stable circ_0000260 knockdown was conducted using shRNA packaged into lentiviral vector to explore the role of circ_0000260 in vivo. The result showed that circ_0000260 knockdown significantly suppressed tumor volume and tumor weight. Meanwhile, with the presence of CDDP and circ_0000260 knockdown, tumor volume and tumor weight were further weakened compared to CDDP presence alone (Figure 8A and B), suggesting that circ_0000260 knockdown could promote CDDP chemosensitivity in vivo. In the removed tumor tissues, we detected that the expression of circ 0000260 was notably decreased in the sh-circtransfected group or CDDP-treated group, while the expression of miR-129-5p opposite to circ_0000260 expression (Figure 8C). Moreover, the expression of MMP11 at both mRNA and protein levels was declined in the sh-circtransfected group or CDDP-treated group compared to sh$\mathrm{NC}$ group or untreated group, respectively (Figure $8 \mathrm{D}$ and E). These data suggested that circ_0000260 targeted the miR129-5p/MMP11 axis to regulate CDDP chemosensitivity in vivo.

\section{Discussion}

The application of multidisciplinary treatment of GAC contributes to the improvement of its prognosis, and CDDP plays a crucial role in this treatment modality for advanced GAC. ${ }^{17}$ Previous studies have reported various factors related to CDDP chemoresistance, such as metastasisrelated genes, fibroblasts-derived IL-8 and non-coding RNAs. ${ }^{18-20}$ In our study, circ_0000260 was monitored to be upregulated in GAC tissues and serum-derived exosomes. Besides, the abundance of circ_0000260 was higher in CDDP-resistant GAC tissues than that in CDDP-sensitive GAC tissues, hinting that circ_0000260 dysregulation might be associated with CDDP chemoresistance. Functional experiments revealed that circ_0000260 knockdown promoted sensitivity of HGC27/CDDP and AGS/CDDP cells 
A
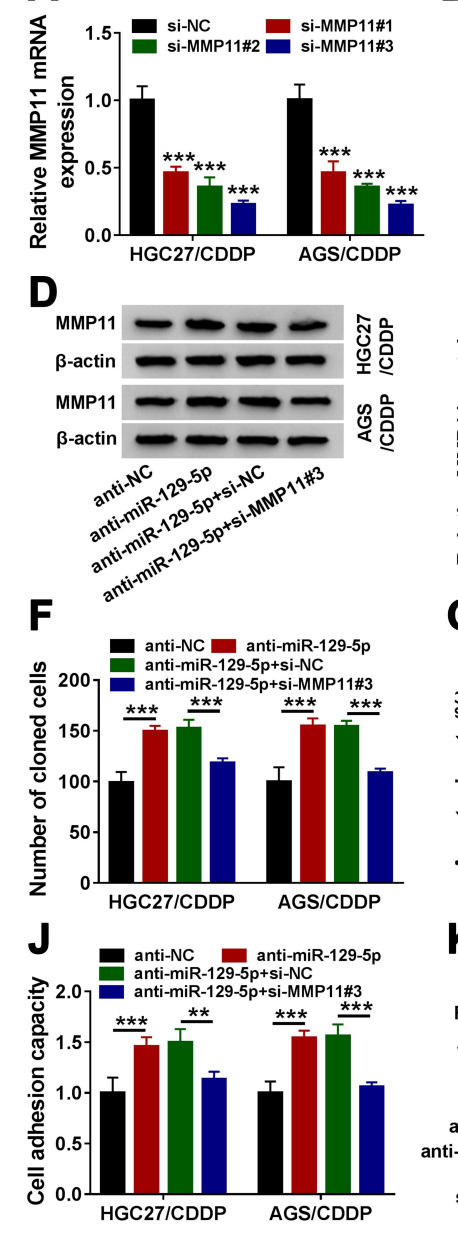

D

anti-NC anti-miR-129-5p

${ }^{200}$ anti-miR-129-5p+si-MMP11\#3

G
B
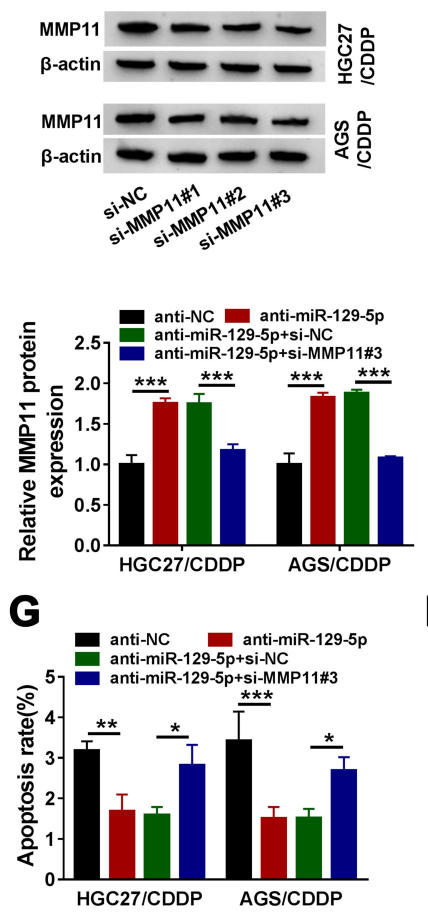

$\mathbf{K}$

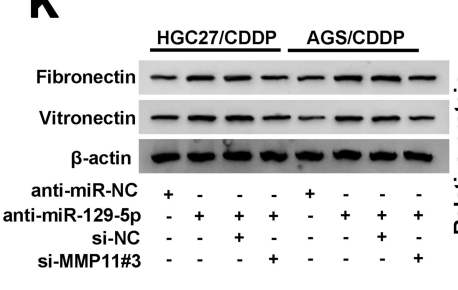

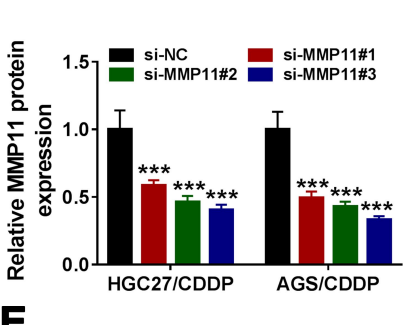

E

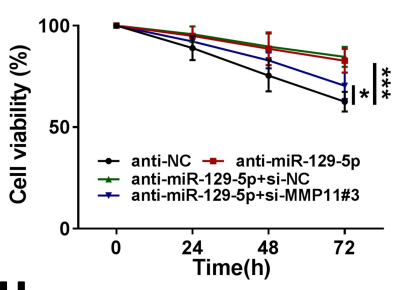

H

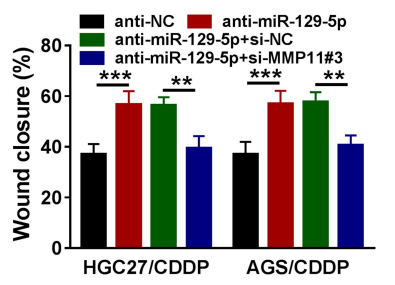

HGC27/CDDP

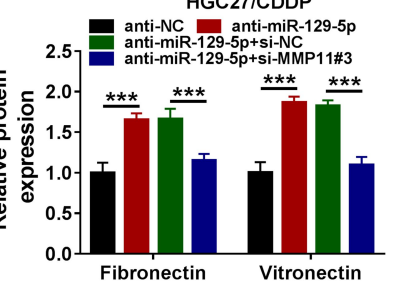

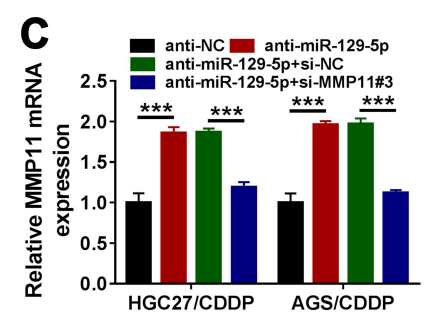

AGS/CDDP

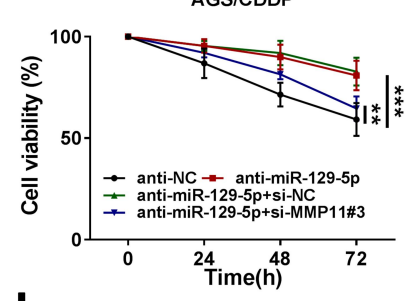

I
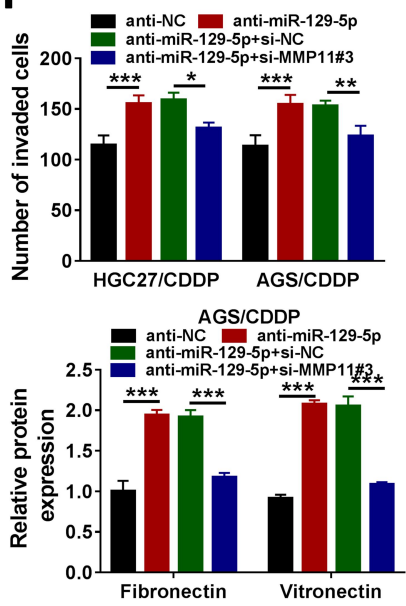

Figure 6 MiR-129-5p regulated CDDP chemoresistance by targeting MMPII. (A and B) The efficiency of siRNA targeting MMPII was examined using QRT-PCR and Western blot. HGC27/CDDP and AGS/CDDP cells were then transfected with anti-miR-I29-5p, anti-NC, anti-miR-I29-5p+si-MMPII or anti-miR-I29-5p+si-NC. (C and D) The expression of MMPII in these cells was detected by qRT-PCR and Western blot. In these indicated cells, (E) cell proliferation, (F) colony formation, (G) cell apoptosis, $(\mathbf{H})$ cell migration, (I) cell invasion and $(\mathbf{J})$ cell adhesion were assessed by CCK-8 assay, colony formation assay, flow cytometry assay, scratch assay, transwell assay and cell adhesion assay, respectively. (K) The expression of fibronectin and vitronectin was quantified by Western blot. $* P<0.05, * * P<0.0 \mathrm{I}, * * * P<0.00 \mathrm{I}$.

A

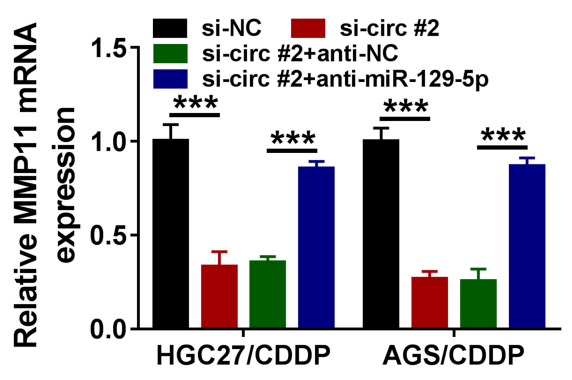

B

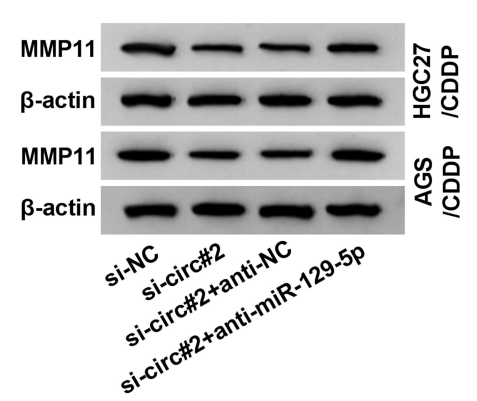

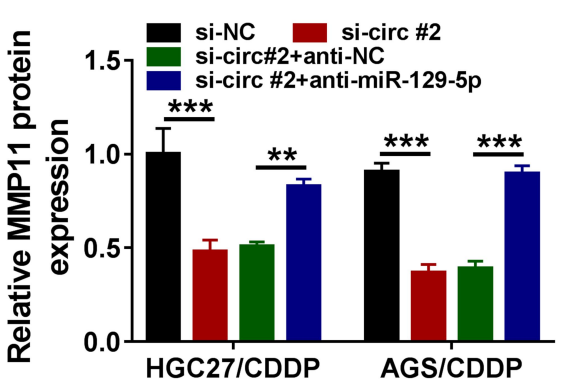

Figure 7 Circ_0000260 positively regulated MMPII expression by targeting miR-I29-5p. (A and B) The expression of circ_0000260 in HGC27/CDDP and AGS/CDDP cells transfected with si-circ\#2, si-NC, si-circ\#2+anti-miR-129-5p or si-circ\#2+anti-NC was detected by qRT-PCR and Western blot. $* * P<0.01$, $* * * P<0.001$.

response to CDDP, thereby suppressing cell proliferation, colony formation, migration, invasion and cell adhesion. Circ_0000260 is produced from SMC3 (NM_005445) mRNA, which is presented to be highly regulated in GAC
(STAD) tissues relative to normal tissues through GEPIA database. The carcinogenic effects of SMC3 were demonstrated in lung cancer, ${ }^{21}$ hinting that SMC3 was closely associated with the progression of multiple cancers. Here, 

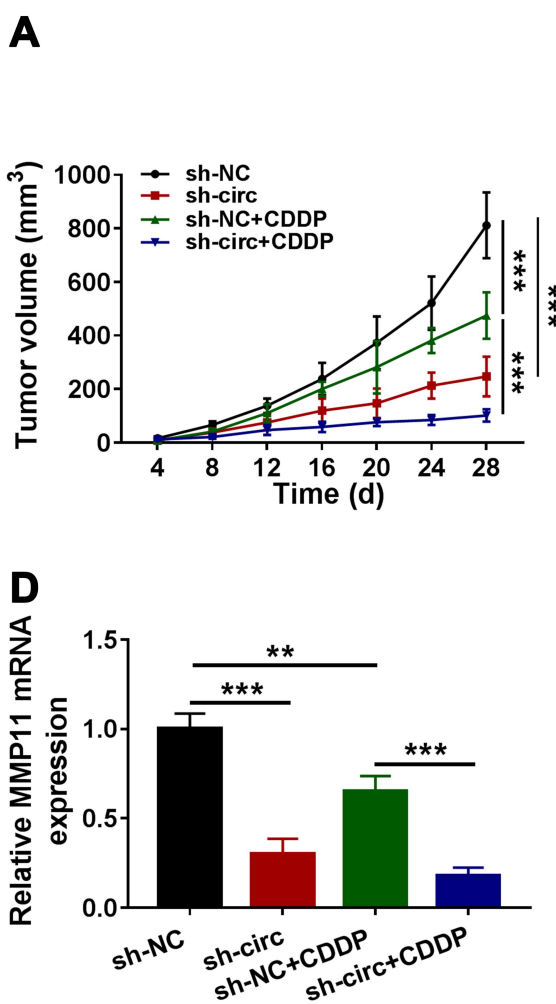
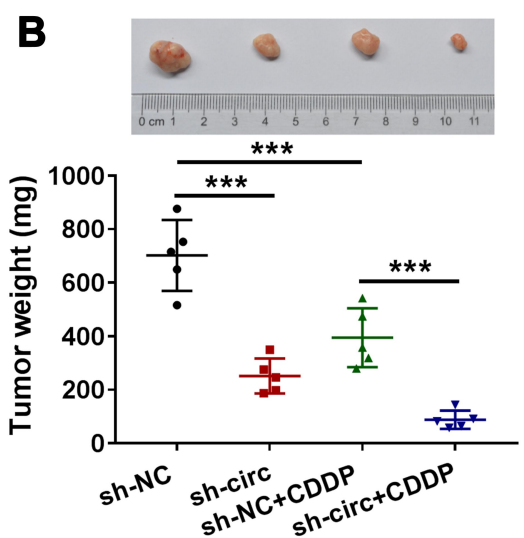

$\mathbf{E}$

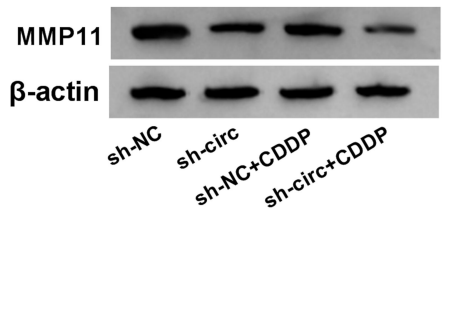

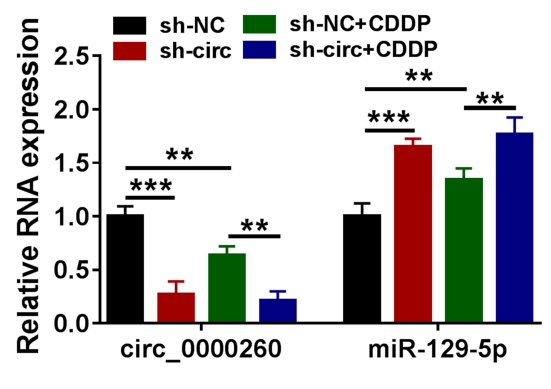

\section{C}

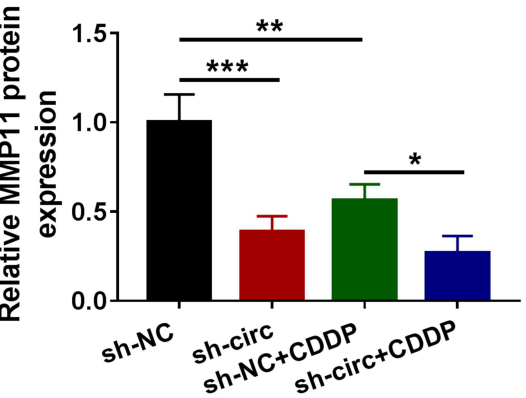

Figure 8 Circ_0000260 knockdown inhibited CDDP chemoresistance during tumor growth in vivo. (A) Tumor volume was recorded every 4 days. (B) Tumor weight was measured at 28 days post-injection. (C) The expression of circ_0000260 and miR-129-5p in removed tumor tissues from different groups was measured by qRT-PCR. (D and E) The expression of MMPII in removed tumor tissues from different groups was measured by qRT-PCR and Western blot. $* P<0.05$, $* * P<0.0 \mathrm{I}$, $* * * P<0.00 \mathrm{I}$.

\section{Nucleus}

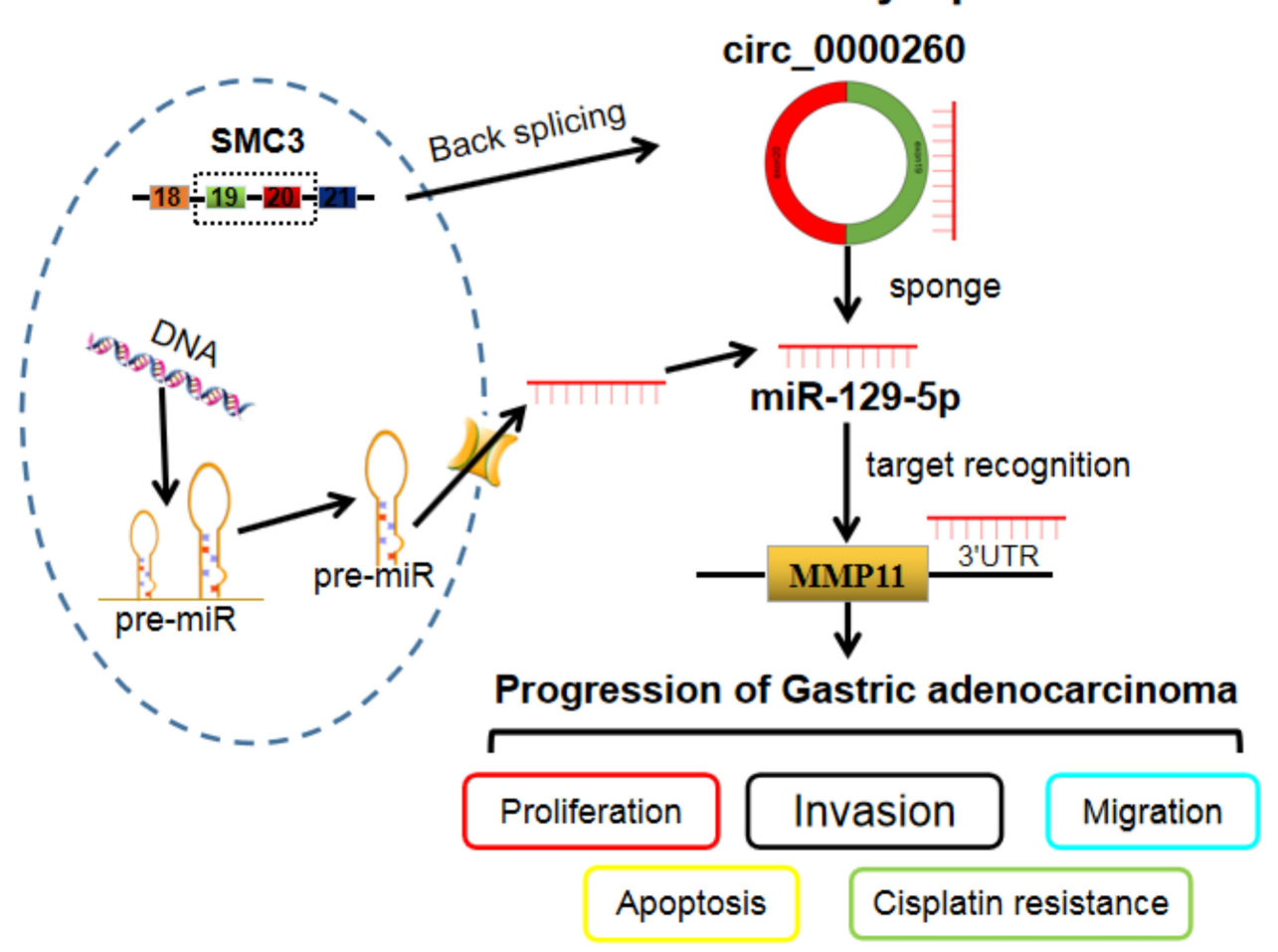

Figure 9 Circ_0000260 functioned as miR-129-5p sponges to enrich the expression of MMPII, thus regulating the progression of GAC. 
we reported that circ_0000260, a splicing form of SMC3, was also implicated in GAC progression. Interestingly, a recent study held a view that circ_0000260 (circSMC3) knockdown depleted the proliferation and motility of gastric cancer cells though other regulatory networks. Both this finding and our data consistently highlighted the cancerpromoting role of circ_0000260 in gastric cancer.

Mechanism analysis claimed that miR-129-5p was a downstream target of circ_0000260. MiR-129-5p was a wellrecognized tumor suppressor in gastric cancer, and its restoration inhibited proliferation, migration, invasion, as well as glycolysis in gastric cancer cells. ${ }^{22,23}$ Meanwhile, miR-1295 p overexpression was documented to reduce CDDP chemoresistance of non-small cell lung cancer, ${ }^{24}$ hinting that miR-129$5 \mathrm{p}$ could regulate CDDP chemoresistance. However, no evidence supported its role in gastric cancer. In our data, miR-129$5 p$ expression was weaker in CDDP-resistant GAC tissues compared with that in CDDP-sensitive GAC tissues. Functional analyses displayed that miR-129-5p inhibition weakened GAC cell chemosensitivity to CDDP and promoted proliferation, metastasis and adhesion of CDDP-resistant GAC cells. Besides, miR-129-5p inhibition also reversed the effects of circ_0000260 knockdown, suggesting that circ_0000260 regulated CDDP chemoresistance by suppressing miR-129-5p.

Further exploration concluded that MMP11 was a target of miR-129-5p. It was reported that MMP11 downregulation attenuated gastric cancer cell proliferative activity and invasive potential. ${ }^{15,25}$ Besides, MMP11 was identified to be a target of miR-139, and miR-139 blocked the progression and metastasis of gastric cancer by targeting MMP11. ${ }^{26}$ Their data also showed that MMP11 expression was increased in exosomes derived from plasma of gastric cancer patients. ${ }^{26}$ Moreover, the expression of MMP11 was aberrantly higher in multidrug resistant-hepatocellular carcinoma cells, and promoted MMP11 strengthened resistance of cells to chemotherapeutic drugs. ${ }^{27}$ In our data, we noticed that the expression of MMP11 was enhanced in CDDP-resistant GAC tissues, and MMP11 functioned as an oncogene to abolish the effects of miR-129$5 \mathrm{p}$, promoted CDDP chemoresistance and triggered CDDPresistant GAC cell malignant behaviors.

\section{Conclusion}

In summary, these studies demonstrated that circ_0000260 knockdown suppressed CDDP chemoresistance and thus blocked CDDP-resistant cell malignant behaviors, including proliferation, migration, invasion and adhesion, by modulating the expression of MMP11 via targeting miR-129-5p (Figure 9). Our findings partly uncovered the role of circ_0000260 and provided further insights to understand the mechanism of chemoresistance in GAC. However, this study is only a preliminary study of the role of circ_0000260 in GAC, and further extensive in-depth studies, such as energy metabolism, should be performed to explore its function. Besides, this study mainly determines the role of circ_0000260 by siRNA-mediated knockdown, and circ_0000260 overexpression should also be conducted to validate its role.

\section{Funding}

There is no funding to report.

\section{Disclosure}

The authors declare that they have no conflicts of interest.

\section{References}

1. Siegel R, Naishadham D, Jemal A. Cancer statistics, 2012. $C A$ Cancer J Clin. 2012;62(1):10-29. doi:10.3322/caac.20138

2. Gu J, Li Y, Fan L, et al. Identification of aberrantly expressed long non-coding RNAs in stomach adenocarcinoma. Oncotarget. 2017;8 (30):49201-49216. doi:10.18632/oncotarget.17329

3. Banks M, Graham D, Jansen M, et al. British Society of Gastroenterology guidelines on the diagnosis and management of patients at risk of gastric adenocarcinoma. Gut. 2019;68 (9):1545-1575. doi:10.1136/gutjnl-2018-318126

4. Cunningham D, Allum WH, Stenning SP, et al. Perioperative chemotherapy versus surgery alone for resectable gastroesophageal cancer. $N \mathrm{Engl}$ $J$ Med. 2006;355(1):11-20. doi:10.1056/NEJMoa055531

5. Cheng S, Mao Q, Dong Y, et al. GNB2L1 and its O-GlcNAcylation regulates metastasis via modulating epithelial-mesenchymal transition in the chemoresistance of gastric cancer. PLoS One. 2017;12(8): e0182696. doi:10.1371/journal.pone.0182696

6. Shao Y, Li J, Lu R, et al. Global circular RNA expression profile of human gastric cancer and its clinical significance. Cancer Med. 2017;6(6):1173-1180. doi:10.1002/cam4.1055

7. Liu X, Cai H, Sheng W, Huang H, Long Z, Wang Y. microRNAs expression profile related with response to preoperative radiochemotherapy in patients with locally advanced gastric cancer. $B M C$ Cancer. 2018;18(1):1048. doi:10.1186/s12885-018-4967-4

8. Zhang X, Wang S, Wang H, et al. Circular RNA circNRIP1 acts as a microRNA-149-5p sponge to promote gastric cancer progression via the AKT1/mTOR pathway. Mol Cancer. 2019;18(1):20. doi:10.1186/ s12943-018-0935-5

9. Wei J, Wei W, Xu H, et al. Circular RNA hsa_circRNA_102958 may serve as a diagnostic marker for gastric cancer. Cancer Biomark. 2020;27(2):139-145. doi:10.3233/CBM-182029

10. Huang X, Li Z, Zhang Q, et al. Circular RNA AKT3 upregulates PIK3R1 to enhance cisplatin resistance in gastric cancer via miR-198 suppression. Mol Cancer. 2019;18(1):71. doi:10.1186/s12943-019-0969-3 
11. Liu YY, Zhang LY, Du WZ. Circular RNA circ-PVT1 contributes to paclitaxel resistance of gastric cancer cells through the regulation of ZEB1 expression by sponging miR-124-3p. Biosci Rep. 2019;39(12). doi:10.1042/BSR20193045

12. Jiang Z, Wang H, Li Y, et al. MiR-129-5p is down-regulated and involved in migration and invasion of gastric cancer cells by targeting interleukin-8. Neoplasma. 2016;63(5):673-680. doi:10.4149/ neo_2016_503

13. Wang Q, Yu J. MiR-129-5p suppresses gastric cancer cell invasion and proliferation by inhibiting COL1A1. Biochem Cell Biol. 2018;96 (1):19-25. doi:10.1139/bcb-2016-0254

14. Gu S, Jin L, Zhang F, Sarnow P, Kay MA. Biological basis for restriction of microRNA targets to the $3^{\prime}$ untranslated region in mammalian mRNAs. Nat Struct Mol Biol. 2009;16(2):144-150. doi:10.1038/nsmb. 1552

15. Kou YB, Zhang SY, Zhao BL, Ding R, Liu H, Li S. Knockdown of MMP11 inhibits proliferation and invasion of gastric cancer cells. Int J Immunopathol Pharmacol. 2013;26(2):361-370. doi:10.1177/ 039463201302600209

16. Barr MP, Gray SG, Hoffmann AC, et al. Generation and characterisation of cisplatin-resistant non-small cell lung cancer cell lines displaying a stem-like signature. PLoS One. 2013;8(1):e54193. doi:10.1371/journal.pone.0054193

17. Japanese Gastric Cancer A. Japanese gastric cancer treatment guidelines 2014 (ver. 4). Gastric Cancer. 2017;20(1):1-19. doi:10.1007/ s10120-016-0622-4.

18. Nishiguchi Y, Oue N, Fujiwara-Tani R, et al. Role of metastasis-related genes in cisplatin chemoresistance in gastric cancer. Int J Mol Sci. 2019;21(1):254. doi:10.3390/ijms21010254

19. Zhai J, Shen J, Xie G, et al. Cancer-associated fibroblasts-derived IL-8 mediates resistance to cisplatin in human gastric cancer. Cancer Lett. 2019;454:37-43. doi:10.1016/j.canlet.2019.04.002
20. Chen C, Tang X, Liu Y, Zhu J, Liu J. Induction/reversal of drug resistance in gastric cancer by non-coding RNAs (Review). Int J Oncol. 2019;54(5):1511-1524. doi:10.3892/ijo.2019.4751

21. Wang D, Wang L, Zhang Y, Zhao Y, Chen G. Hydrogen gas inhibits lung cancer progression through targeting SMC3. Biomed Pharmacother. 2018;104:788-797. doi:10.1016/j.biopha.2018.05.055

22. Liu Q, Jiang J, Fu Y, Liu T, Yu Y, Zhang X. MiR-129-5p functions as a tumor suppressor in gastric cancer progression through targeting ADAM9. Biomed Pharmacother. 2018;105:420-427. doi:10.1016/j. biopha.2018.05.105

23. Chen D, Wang H, Chen J, et al. MicroRNA-129-5p regulates glycolysis and cell proliferation by targeting the glucose transporter SLC2A3 in gastric cancer cells. Front Pharmacol. 2018;9(502). doi:10.3389/fphar.2018.00502

24. Ma Z, Cai H, Zhang Y, Chang L, Cui Y. MiR-129-5p inhibits non-small cell lung cancer cell stemness and chemoresistance through targeting DLK1. Biochem Biophys Res Commun. 2017;490 (2):309-316. doi:10.1016/j.bbrc.2017.06.041

25. Deng H, Guo RF, Li WM, Zhao M, Lu YY. Matrix metalloproteinase 11 depletion inhibits cell proliferation in gastric cancer cells. Biochem Biophys Res Commun. 2005;326(2):274-281. doi:10.1016/ j.bbrc.2004.11.027

26. $\mathrm{Xu} \mathrm{G}$, Zhang $\mathrm{B}$, Ye $\mathrm{J}$, et al. Exosomal miRNA-139 in cancer-associated fibroblasts inhibits gastric cancer progression by repressing MMP11 expression. Int $J$ Biol Sci. 2019;15 (11):2320-2329. doi:10.7150/ijbs.33750

27. Jia L, Xu H, Zhao Y, Jiang L, Yu J, Zhang J. Expression of CD147 mediates tumor cells invasion and multidrug resistance in hepatocellular carcinoma. Cancer Invest. 2008;26(10):977-983. doi:10.1080/ 07357900802072723

\section{Publish your work in this journal}

Cancer Management and Research is an international, peer-reviewed open access journal focusing on cancer research and the optimal use of preventative and integrated treatment interventions to achieve improved outcomes, enhanced survival and quality of life for the cancer patient.
The manuscript management system is completely online and includes a very quick and fair peer-review system, which is all easy to use. Visit http://www.dovepress.com/testimonials.php to read real quotes from published authors. 